\title{
On Generalizations of the KMS-Boundary Condition
}

\author{
J. S. Cohen ${ }^{1}$, H. A. M. Daniëls ${ }^{2}$, and M. Winnink ${ }^{2}$ \\ 1 Institute for Theoretical Physics, University of Amsterdam, Amsterdam, The Netherlands \\ 2 Institute for Theoretical Physics, University of Groningen, Groningen, The Netherlands
}

\begin{abstract}
We investigate the possibility to generalize the KMS-boundary condition for a thermodynamical system by following essentially the same procedure that for a finite system would amount to choosing a certain class of more general density functions on phase space (or density matrices) than the ones corresponding to the canonical or grand-canonical ensemble.
\end{abstract}

\section{Introduction}

Gibbs states of thermodynamical systems in classical physics are described by means of conditional probabilities that are related to the energy of interaction of the configurations of finite systems with the outside world by means of an exponential function.

In [1] states of classical systems are considered where the above mentioned exponential function is replaced by a set of more general (albeit invertible) functions. These states are called regular conditional equilibrium (CE) states. A subclass of these states can, under suitable conditions, be shown to be Gibbs states [1]. The CE states are to be viewed as states obtained as thermodynamical limits of microcanonical states for finite systems. On the other hand Gibbs states are thermodynamical limits of canonical states for finite systems with specified boundary conditions (or convex combinations thereof) (cf. [2]).

Once one can show as in [1] that, under suitable conditions, regular CE states are Gibbs states, then one has made statements about the "equivalence of ensembles." Loosely speaking one can say that the exponential function, representing the Gibbs character of the state, is the thermodynamical significant object.

For quantum systems, under suitable conditions on the way one performs the thermodynamic limit on the finite volume correlation functions and the dynamics, one obtains the well-known KMS-boundary condition [3]. The KMS-boundary condition as introduced in [3] reads:

$$
\int_{-\infty}^{\infty} \omega\left(A \alpha_{t}(B)\right) f(t-i \beta) d t=\int_{-\infty}^{\infty} \omega\left(\alpha_{t}(B) A\right) f(t) d t
$$


with

$$
t \rightarrow \omega\left(A \alpha_{t} B\right) \text { a continuous function on } \mathbb{R}^{1} .
$$

Here $\left\{\alpha_{t}\right\}_{t \in \mathbb{R}^{1}}$ is a one-parameter group of automorphisms of a $C^{*}$-algebra $\mathfrak{H}, f$ a function with its Fourier transform $\hat{f} \in D$ and $\omega$ a state on $\mathfrak{U}$.

By Fourier transforming (1) we obtain

$$
\hat{F}_{A B}\left(\tilde{\hat{f}} e^{-\beta \varepsilon}\right)=\hat{F}_{B A}(\hat{f}),
$$

where $\hat{F}_{A B}$ is the Fourier transform of the distribution determined by the function $t \rightarrow \omega\left(A \alpha_{t}(B)\right), \breve{f}(x)=f(-x)$. From (2) one sees that the Gibbs-like nature of the thermodynamical KMS state $\omega$ is reflected in the appearance of $e^{-\beta \varepsilon}$ as a multiplier in the sense of distribution theory.

Regular CE states in [1] are obtained by replacing the exponential weightfunction appearing in the conditional probabilities for configurations of finite systems by another invertible function. In this paper we intend to study to what extent something similar for thermodynamical systems as a whole, can be done. More precisely we are going to study the following equation:

$$
\hat{F}_{A B}\left(\check{\hat{f}}_{\phi}\right)=\hat{F}_{B A}(\hat{f}) \text {, }
$$

or equivalently

$$
\int_{-\infty}^{\infty} \omega\left(A \alpha_{t} B\right) f_{\phi}(t) d t=\int_{-\infty}^{\infty} \omega\left(\alpha_{t}(B) A\right) f(t) d t,
$$

where $t \rightarrow \omega\left(A \alpha_{t} B\right)$ is continuous for all $A$ and $B, \hat{f}_{\phi}=\hat{f} \cdot \phi$ and $\phi$ is a $C^{\infty}$-function from $\mathbb{R}$ into $\mathbb{C}$ such that $\phi(x)-1$ has no zeros except at $x=0$. States satisfying condition (4) with a given $\phi$ are called $\phi$-KMS states. If $\phi(x)=e^{\beta x}$ (3) and (4) are nothing but the KMS-boundary condition.

Equation (3) and (4) are also obtained as an intermediate step in deriving the KMS-boundary condition for a system with a strongly continuous dynamics (i.e. $\left.\left\|\alpha_{t}(A)-A\right\| \begin{array}{r}\rightarrow 00 \\ t \rightarrow 0\end{array}\right)$ from stability properties of the system, sufficiently fast decrease properties of the time-correlation functions up to fourth order and the assumption that the spectrum of the generator of time translations fills $\mathbb{R}^{1}$. By making use of the cluster-properties of the two-point function it is then shown that $\phi$ is an exponential function $[4,5]$ (cf. also $[6,7]$ ). The cluster-properties used in the proof restrict its applicability to a one-phase region since the obtained KMS state is extremal.

We will find, without further assumptions, that for quantum lattice systems, $\phi$ satisfying (3) and (4) is necessarily an exponential function on the spectrum of the generator of time-translations (the Liouville-operator). Generalizing to continuous quantum systems we find that for such systems with a sufficiently regular dynamics and a compactness assumption on the set of states $\omega$ that obey (3) and (4) $\phi$ is also an exponential function on the spectrum of the generator of timetranslations (Theorem 1, Sect. 3). Hence under these conditions (3) and (4) admit nothing but KMS states as solutions. 
We will furthermore show that in the classical limit (3) coincides with the classical KMS condition for some (possibly negative) temperature.

Since the generalized KMS condition as proposed by J. V. Pulé in [8] is a particular case of the condition formulated in (3) and (4) (see Sect. 4) all our conclusions apply also to the condition formulated in [8].

\section{Invariance, Separating Character and the Modular Group of a $\phi$-KMS State}

We start with the most simple case: a finite Quantum spin system. Some of the features discovered here will hold true in general. Let $\mathfrak{U}=\mathbb{C}^{n \times n}$ the algebra of $n \times n$ complex matrices. The one parameter group of automorphisms $\alpha_{t}$ is given by $\alpha_{t}(A)$ $=U_{t} A U_{-t}$ where $U_{t}$ is unitary. The infinitesimal generator of $U_{t}$ is denoted by $H$. We write $H=\sum_{l=1}^{n} \varepsilon_{l} \cdot E_{l}$ where $E_{l}$ are orthogonal rank one projectors and $\varepsilon_{l}$ the eigenvalues of $H$. A state $\omega$ is defined by a positive matrix $\varrho$ with trace $\varrho=1$. $\omega$ satisfies the $\phi$-KMS equation if

$$
\int_{-\infty}^{\infty} \operatorname{Tr}\left(\varrho A \alpha_{t}(B)\right) f_{\phi}(t) d t=\int_{-\infty}^{\infty} \operatorname{Tr}\left(\varrho \alpha_{t}(B) A\right) f(t) d t .
$$

Lemma 1. $\varrho$ determines a $\phi$-KMS state if and only if $\varrho=\sum_{l=1}^{n} \varrho_{l} E_{l}$ and $\varrho_{i} / \varrho_{j}$
$=\phi\left(\varepsilon_{j}-\varepsilon_{i}\right)$. Proof. The integrals $\int_{-\infty}^{\infty} f(t) \alpha_{t}(B) d t$ and $\int_{-\infty}^{\infty} f_{\phi}(t) \alpha_{t}(B) d t$ are both well defined. One
computes:

$$
\int_{-\infty}^{\infty} f(t) \alpha_{t}(B) d t=\sum_{k=1}^{n} \sum_{l=1}^{n} \hat{f}\left(\varepsilon_{l}-\varepsilon_{k}\right) E_{k} B E_{l}
$$

and

$$
\int_{-\infty}^{\infty} f_{\phi}(t) \alpha_{t}(B) d t=\sum_{k=1}^{n} \sum_{l=1}^{n} \phi\left(\varepsilon_{l}-\varepsilon_{k}\right) \hat{f}\left(\varepsilon_{l}-\varepsilon_{k}\right) E_{k} B E_{l} .
$$

If we choose $\hat{f}=1$ on $\Sigma:=\left\{\varepsilon_{l}-\varepsilon_{k} \mid l, k=1,2, \ldots, n\right\}$ we see that (5) implies

$$
\operatorname{Tr}(\varrho A \Gamma(B))=\operatorname{Tr}(\varrho B A),
$$

where $\Gamma: \mathfrak{U} \rightarrow \mathfrak{U}$ is defined by:

$$
\Gamma(X)=\sum_{k=1}^{n} \sum_{l=1}^{n} \phi\left(\varepsilon_{l}-\varepsilon_{k}\right) E_{k} X E_{l} .
$$

(6) is equivalent with

$$
\Gamma(X) \cdot \varrho=\varrho X \quad \text { for all } X \in \mathfrak{U}
$$

and from this one easily finds $\varrho=\sum_{l=1}^{n} \varrho_{l} E_{l}\left(\Gamma\left(E_{l}\right)=E_{l}\right)$ and $\varrho_{i} / \varrho_{j}=\phi\left(\varepsilon_{j}-\varepsilon_{i}\right)$. It is easy to see that this is also sufficient. 
Remark 1. It follows from this lemma that the density matrix $\varrho$ is a function of the Hamiltonian and $\omega$ is therefore invariant. Furthermore $\varrho$ is invertible and $\omega$ is therefore separating. Note that also $\phi(x) \in \mathbb{R}$ and $\phi(x) \cdot \phi(-x)=1$ for $x \in \Sigma=\left\{\varepsilon_{i}-\varepsilon_{j}\right.$; $i, j \in(1 \ldots n)\}$. These properties turn out to be true in general. We now consider the general case and prove that every $\phi$-KMS state is invariant and separating.

Lemma 2. If $\omega$ is a $\phi$-KMS state then $\omega$ is invariant for $\alpha_{t}$.

Proof. For $B \in \mathfrak{U}$ we write

$$
G_{B}(t)=\omega\left(\alpha_{t}(B)\right) .
$$

We consider $G_{B}$ as a distribution on $Z(Z=\hat{D})$. If we take $A=\mathbb{1}$ in (4) we get

$$
\left(G_{B}, f_{\phi}\right)=\left(G_{B}, f\right) \text {. }
$$

Hence

And therefore:

$$
\left(\hat{G}_{B}, \hat{f}_{\phi}\right)=\left(\hat{G}_{B}, \hat{f}\right)
$$

$$
\left(\hat{G}_{B}, f(\phi-1)\right)=0 \text { for all } f \in D .
$$

Since $\phi(x)-1$ has no zeros except at the origin, it follows that $\hat{G}_{B}$ has its support in 0 . It follows from a standard argument [9a] that $\omega$ is invariant for $\alpha_{t}$.

To proceed further it is convenient to write Eq. (4) in the GNS representation. Let $(\mathscr{H}, \pi, \Omega)$ the GNS triple associated to $\omega$. Since $\omega$ is invariant $\alpha_{t}$ can be implemented by a strongly continuous group of unitaries $U_{t}$ such that $U_{t} \Omega=\Omega$. The generator of $U_{t}$, i.e. the Liouville operator, will be denoted by $L . \Sigma$ is the spectrum of $L$. In the representation $\phi$-KMS reads :

$$
\int_{-\infty}^{\infty}\left(\Omega, \pi(A) U_{t} \pi(B) \Omega\right) f_{\phi}(t) d t=\int_{-\infty}^{\infty}\left(\Omega, \pi(B) U_{-t} \pi(A) \Omega\right) f(t) d t
$$

This equation can be extended to the von Neumann algebra $\pi(\mathfrak{l})^{\prime \prime}$ (cf. [9b])

$$
\int_{-\infty}^{\infty}\left(\Omega, A U_{t} B \Omega\right) f_{\phi}(t) d t=\int_{-\infty}^{\infty}\left(\Omega, B U_{-t} A \Omega\right) f(t) d t
$$

We can rewrite this equation by defining bounded operators $U_{\phi}$ and $U$ on $\mathscr{H}$ by

$$
\begin{aligned}
& \int_{-\infty}^{\infty}\left(x, U_{t} y\right) f_{\phi}(t) d t=\left(x, U_{\phi} y\right), \\
& \int_{-\infty}^{\infty}\left(x, U_{-t} y\right) f(t) d t=(x, U y) .
\end{aligned}
$$

One computes easily:

$$
U_{\phi}=(\hat{f} \phi)^{2}(L) \text { and } U=\hat{f}(L) .
$$

Now (7) can be rewritten as

$$
\left(A^{*} \Omega,(\hat{f} \phi)^{2}(L) B \Omega\right)=\left(B^{*} \Omega, \hat{f}(L) A \Omega\right)
$$

for all $A, B \in \pi(\mathfrak{H})^{\prime \prime}$. 
Lemma 3. If $\omega$ is $\phi$-KMS then $\Omega$ is separating.

Proof. Suppose $B \Omega=0$, then for all $\hat{f} \in D$

$$
\left(B^{*} \Omega, \hat{f}(L) A \Omega\right)=0 .
$$

We now take $\hat{f}_{n} \in D$ such that $\hat{f}_{n} \rightarrow 1$ (equal to 1 on large intervals) then $\hat{f}_{n}(L) \rightarrow 1$ strongly and therefore

$$
\left(B^{*} \Omega, A \Omega\right)=0 \text { for all } A \text {. }
$$

Hence $B^{*} \Omega=0$ and therefore, by standard arguments [9b], $B=0$.

Lemma 4. If $\omega$ is $\phi$-KMS then:

i) $\phi(x) \in \mathbb{R}, x \in \Sigma$

ii) $\phi(x) \cdot \phi(-x)=1, x \in \Sigma$.

Proof. (8) implies

$$
\left(A^{*} \Omega,(\hat{f} \phi)^{\swarrow}(L) A^{*} \Omega\right) \in \mathbb{R}
$$

for all $A$ when $\hat{f}$ is real. Hence $(\hat{f} \phi)(\lambda) \in \mathbb{R}$ for $\lambda \in \Sigma$ and therefore i) holds. To prove ii) we apply (8) twice to get

$$
\left(B^{*} \Omega, \hat{f}(L) A \Omega\right)=\left(B^{*} \Omega,(\hat{f} \phi \check{\phi})(L) A \Omega\right) .
$$

So

$$
\hat{f}(1-\phi \check{\phi})(L)=0 \text { for all } \hat{f} \in D
$$

and therefore $\phi(x) \cdot \phi(-x)=1$ be the spectral mapping theorem.

Since $\Omega$ is separating one can define the modular operator $\Delta$ corresponding to it. $\Delta$ turns out to be a function of $L$.

Lemma 5. $\Delta^{2}=\check{\phi}(L)$.

Proof. The reasoning is the same as the one followed in [4].

\section{The $\phi$-KMS Condition on Quasi-Local Algebras}

In this section we consider thermodynamical systems which have the following properties :

a) The $C^{*}$-algebra $\mathfrak{U}$ is quasi-local (cf. [10]).

b) The dynamics $\left(\alpha_{t}\right)_{t \in \mathbb{R}}$ is strongly continuous on a $\sigma(\mathfrak{H}, N)$ dense $C^{*}$ subalgebra $\mathfrak{H}_{0} \subset \mathfrak{U} . N$ is the set of locally normal states (cf. [11]).

c) The set of $\phi$-KMS states, $K_{\phi}$, is compact in the weak * topology of $\mathfrak{U}^{*}$.

d) There exists an amenable abelian group $G$, like space translations, which acts on $\mathfrak{U}$ by the automorphisms $\left(\tau_{x}\right)_{x \in G}$ commuting with $\alpha_{t}$. This group $\left(\tau_{x}\right)_{x \in G}$ acts (weakly) asymptotically abelian.

(In particular quantum lattice systems have these properties.)

Lemma 6. Let $\omega \in K_{\phi}$ and suppose $\omega$ is G-invariant. Then $\omega$ is KMS. 
Proof. Let $I_{G}(\mathfrak{U})$ denote the set of $\tau_{x}$-invariant states on $\mathfrak{U}$. We will argue that $\omega$ has a decomposition in extremal invariant $\phi$-KMS states, i.e.

$$
\omega=\int_{\operatorname{Ext}\left(I_{G}(\mathfrak{l}) \cap K_{\phi}\right)} \omega_{\gamma} d \mu(\gamma)
$$

with $\omega_{\gamma}$ extremal invariant.

First note that $K_{\phi} \cap I_{G}(\mathfrak{U})$ is a simplex. Due to assumption c) we find that $K_{\phi} \cap I_{G}(\mathfrak{U})$ is metrizable and $\operatorname{Ext}\left(K_{\phi} \cap I_{G}(\mathfrak{U})\right)$ is a $G_{\delta}$. (These results can be found in [12].) Therefore the decomposition (9) can be made. Since $\left(\tau_{x}\right)_{x \in G}$ is weakly asymptotically abelian (i.e. $\left.\omega\left(\left[A, \tau_{x}(B)\right]\right) \rightarrow 0, x \rightarrow \infty\right)$ we can apply Theorem 2.3 of [13] and Lemma $4.7^{\prime}$ of [12] to conclude that $I_{G}(\mathfrak{U})$ is a face of $K_{\phi} \cap I_{G}(\mathfrak{U})$ and therefore the decomposition of $\omega$ is into extremal invariant states. Hence $\omega_{\gamma}$ is weakly clustering for $\left(\tau_{x}\right)_{x \in G}$, i.e.

$$
\eta_{x}\left(\omega_{\gamma}\left(A \tau_{x}(B)\right)-\omega_{\gamma}(A) \omega_{\gamma}(B)\right)=0,
$$

where $\eta_{x}$ is a mean over $G$. Let $\left(\mathscr{H}_{\gamma}, \pi_{\gamma}, \Omega_{\gamma}\right)$ be the GNS triple for $\omega_{\gamma}$. We know from Lemma 4 that

$$
\Delta_{\gamma}^{2}=\breve{\phi}\left(L_{\gamma}\right),
$$

where $L_{\gamma}$ is the Liouville operator of $\alpha_{t}$ in this representation. We have the following groups of automorphisms of the von Neumann algebra $\pi_{\gamma}(\mathfrak{U})^{\prime \prime}$.

1) Time evolution $\alpha_{t}^{\gamma}(A)=U_{t}^{\gamma} A U_{-t}^{\gamma}, U_{t}^{\gamma}=\exp i t L_{\gamma}$.

2) The modular group $A \rightarrow \Delta_{\gamma}^{i s} A \Delta_{\gamma}^{-i s}$.

3) The translation group $\tau_{x}(A)=T_{x} A T_{-x}$.

We define the 2-parameter group

$$
W_{t, s}(A)=U_{t}^{\gamma} \Delta_{\gamma}^{i s} A \Delta_{\gamma}^{-i s} U_{-t}^{\gamma}, A \in \pi_{\gamma}(\mathfrak{H})^{\prime \prime} .
$$

There exists a $C^{*}$-algebra $C_{0} \subset \pi_{\gamma}(\mathfrak{l})^{\prime \prime}$ such that

i) $C_{0}$ is $\sigma\left(\pi_{\gamma}(\mathfrak{U})^{\prime \prime}, \pi_{\gamma}(\mathfrak{U})_{*}^{\prime \prime}\right)$ dense in $\pi_{\gamma}(\mathfrak{H})^{\prime \prime}$,

ii) $W_{t, s}$ is strongly continuous on $C_{0}$.

To see this define $\tilde{\mathfrak{U}}_{0}=\left\{A_{f} \mid \hat{f} \in D, A \in \mathfrak{U}_{0}\right\}$ and $A_{f}=\int_{-\infty}^{\infty} \alpha_{t}(A) f(t) d t$.

Take $C_{0}$ the $C^{*}$-closure of $\pi_{\gamma}\left(\tilde{\mathfrak{U}}_{0}\right)$. Every $A \in \pi_{\gamma}\left(\tilde{\mathfrak{U}}_{0}\right)$ is anylytic for both $\alpha_{t}^{\gamma}$ and the modular group, therefore $W_{t, s}$ is strongly continuous on $\pi_{\gamma}\left(\tilde{\mathfrak{U}}_{0}\right)$, hence on $C_{0}$, proving ii). Since $\mathfrak{U}_{0}$ is $\sigma(\mathfrak{U}, N)$ dense in $\mathfrak{U}$ and $\pi_{\gamma}(\mathfrak{H})_{*}^{\prime \prime} \subset N$ (cf. [12]), it follows that i) is true. The group $W_{t, s}$ commutes with $\tau_{x}$. We now apply Theorem 3.4 of [14] to conclude that the spectrum of $W_{t, s}$ is a subgroup of $\mathbb{R}^{2}$.

On the other hand the spectrum of $W_{t, s}$ is equal to

$$
\left\{\left(\lambda, \frac{1}{2} \log \check{\phi}(\lambda)\right) \mid \lambda \in \Sigma_{\gamma}\right\},
$$

where $\Sigma_{\gamma}$ is the spectrum of $L_{\gamma}$. Hence there exists $\beta_{\gamma} \in \mathbb{R}$ such that

$$
\check{\phi}(\lambda)=e^{\beta_{\gamma} \cdot \lambda}, \quad \lambda \in \Sigma_{\gamma} .
$$

However the set $\Sigma_{\gamma}$ is independent of $\gamma$. This was proved for KMS states in [10]. Theorem A, the same proof applies in our case and therefore:

$$
\check{\phi}(\lambda)=e^{\beta \lambda} \text { on } \Sigma_{\omega} \text {. }
$$


So

$$
\Delta_{\omega}^{2}=e^{\beta L_{\omega}}
$$

and therefore $\omega$ is a KMS state for $\alpha_{t}$.

Theorem 1. Under the assumptions a)-d) every $\omega \in K_{\phi}$ is $K M S$.

Proof. Let $\omega \in K_{\phi}$ and let $\eta$ be a mean over $G$. It follows from Lemma 3 in [15] that there exists a linear map $M_{\eta}: \mathfrak{U}^{*} \rightarrow \mathfrak{U}^{*}$ which projects on the $G$-invariant states. Therefore $\tilde{\omega}:=M_{\eta} \omega$ is $G$ invariant. $M_{\eta} \omega$ is in the weak * closure of the convex hull of the set $\left\{\omega \circ \tau_{x} \mid x \in G\right\}$ and since $K_{\phi}$ is compact $\tilde{\omega}$ is also a $\phi$-KMS state. It follows from the proceding lemma that

$$
\check{\phi}(\lambda)=e^{\beta \lambda} \text { on } \Sigma_{\tilde{\omega}},
$$

but since $\Sigma_{\tilde{\omega}}=\Sigma_{\omega}=$ spectrum of $\alpha_{t}$, we find

$$
\Delta_{\omega}^{2}=e^{\beta L_{\omega}}
$$

and therefore $\omega$ is $\mathrm{KMS}$ for $\alpha_{t}$.

Remark. $\beta=0$ is excluded for a quasi-local algebra that contains infinite von Neumann algebras.

\section{Pulé's $g$-KMS Condition and the Classical Limit}

In this paragraph $\mathfrak{U}$ is a $C^{*}$-algebra of observables, $\alpha_{t}$ a strongly continuous group of * automorphisms. We shall show that every $g$-KMS state in the sense of [8] is also $\phi$-KMS. We start from the following equation of [8]:

$$
\omega\left(\tau_{t A}(B)-B\right)=-i \int_{0}^{t} F f_{A, \tau_{s A}(B)} d s .
$$

Here $\tau_{s A}(B)=\exp (i s A) B \exp (-i s A)$ for $A, B \in \mathfrak{U}$. $f_{A, B}(x)=\omega\left(\alpha_{-i x}(A) B\right)$ for $A$ an analytic element for $\alpha_{t}$. Furthermore $F$ is a distribution in $S^{\prime}$ with compact support and $\hat{F}$ an entire analytic function with no zeros on the imaginary axis except at zero. We take the derivative, with respect to $t$, at both sides of (10) and put $t=0$ :

$$
\omega(B A)=\left(T, f_{A B}\right),
$$

where $T=F+\delta$. For $B$ an entire analytic element for $\alpha_{t}$ with $\omega\left(A \alpha_{z} B\right) \in Z$ there exists a function $\xi_{A B} \in D$ such that:

$$
\omega\left(A \alpha_{z}(B)\right)=\int_{-\infty}^{\infty} e^{-i z t} \xi_{A B}(t) d t .
$$

Now (11) can be rewritten into:

$$
\omega(B A)=\left(T, f_{A B}\right)=\left(T, \mathscr{L} \xi_{A B}\right)=\left(\mathscr{L} T, \xi_{A B}\right)=\int_{-\infty}^{\infty} \hat{T}(i x) \xi_{A B}(x) d x,
$$

where $\mathscr{L} f$ denotes the two-sided Laplace transform of $f$. Clearly $\xi_{A B}$ satisfies

$$
\xi_{A \alpha_{s}(B)}(t)=e^{-i s t} \xi_{A B}(t) \text {. }
$$


Combining this with (12) we get for $\hat{f} \in D$ :

$$
\begin{aligned}
\int_{-\infty}^{\infty} \omega\left(\alpha_{s}(B) A\right) f(s) d s & =\int_{-\infty}^{\infty} \int_{-\infty}^{\infty} \hat{T}(i x) \xi_{A \alpha_{s}(B)}(x) f(s) d x d s \\
& =\int_{-\infty}^{\infty} \int_{-\infty}^{\infty} e^{-i s x} f(s) \hat{T}(i x) \xi_{A B}(x) d x d s \\
& =\int_{-\infty}^{\infty} \hat{T}(i x) \xi_{A B}(x) \hat{f}(x) d x .
\end{aligned}
$$

Define $\phi(x)=\hat{T}(i x)$ then the latter equation is equivalent to

$$
\begin{aligned}
\int_{-\infty}^{\infty} \omega\left(\alpha_{s}(B) A\right) f(s) d s & =\int_{-\infty}^{\infty} \xi_{A B}(x) \hat{f}(x) \phi(x) d x \\
& =\int_{-\infty}^{\infty} \omega\left(A \alpha_{s}(B)\right) f_{\phi}(s) d s,
\end{aligned}
$$

which is the $\phi$-KMS condition. This equation can be extended to all $A, B \in \mathfrak{U}$ because in [8] $\alpha_{t}$ is strongly continuous, and the elements $B$, as we have chosen them, form a dense set in $\mathfrak{U}$.

\section{The Classical Limit}

The generalized KMS condition has no a priori meaning for classical systems. However if we take the classical limit, i.e. $\hbar \rightarrow 0$ in such a way that

$$
\frac{[A, B]}{i \hbar} \rightarrow\{A, B\}
$$

and

$$
\frac{\delta^{(n)}(A)}{\hbar^{n}} \rightarrow\{A, H\}^{(n)},
$$

where $\{A, B\}$ denotes the Poisson bracket and $\{A, H\}^{(n+1)}=\left\{\{A, H\}^{(n)}, H\right\}$ then it turns out that the generalized KMS equation yields the classical KMS condition. An easy way to see this is to start from (11)

$$
\omega(B A)=T \omega\left(\alpha_{-i x}(A) B\right)
$$

or

$$
\omega(B A)-\omega(A B)=T \omega\left(\alpha_{-i x}(A) B-A B\right) .
$$

We divide both sides by $i \hbar$ and let $\hbar \rightarrow 0$. Take $A$ analytic for $\alpha_{t}$ then:

$$
\alpha_{-i x}(A)=\sum_{n=0}^{\infty} \frac{(-i x)^{n}}{n !} \delta^{(n)}(A) .
$$

The right hand side of (13) tends to

$$
\omega(\{B, A\})
$$


whereas the left hand side tends to

$$
-(T, x) \omega(\{A, H\} B) .
$$

So this gives us

$$
\omega(\{A, B\})=(T,(x)) \omega(\{A, H\} B),
$$

which is the classical KMS condition with inverse temperature [16]

$$
\beta=(T, x) .
$$

With $\phi(x)=\hat{T}(i x)$ one easily computes

$$
\beta=\phi^{\prime}(0) .
$$

Remark 2. For a finite classical system one would now expect $\varrho(x)=e^{-\beta H(x)}$ as the only solutions of the $\phi$-KMS equation. This is indeed the case. For a finite classical system $\phi$-KMS should be (in view of Lemma 1)

$$
\frac{\varrho(x)}{\varrho(y)}=\phi(H(x)-H(y)) \text {. }
$$

It is easy to see that this implies $\phi(x)=e^{\beta x}$ on the range of $H$.

\section{References}

1. Aizenman, M., Goldstein, S., Lebowitz, J.L.: Conditional equilibrium and equivalence of microcanonical and grandcanonical ensembles in the thermodynamical limit. Commun. Math. Phys. 62, 279-302 (1978)

2. Ruelle, D.: Thermodynamic formalism, Chap. 1. Encyclopedia of Mathematics, Vol. 5. Reading, MA: Addison-Wesley 1978

3. Haag, R., Hugenholtz, N.M., Winnink, M.: On the equilibrium states in quantum statistical mechanics. Commun. Math. Phys. 5, 215-236 (1967)

4. Hoekman, F.: On stability and symmetries in quantum statistical mechanics. Thesis, Groningen (1977)

5. Bratteli, O., Kastler, D. : Relaxing the clustering condition in the derivation of the KMS-property. Commun. Math. Phys. 46, 37-42 (1976)

6. Haag, R., Kastler, D., Trych-Pohlmeyer, E.B.: Stability and equilibrium states. Commun. Math. Phys. 38, 173-193 (1974)

7. Bratteli, O., Robinson, D.W.: Operator algebra's and quantum statistical mechanics. II. Chap. 5, Sect. 4. Berlin, Heidelberg, New York: Springer 1981

8. Pulé, J.V.: A unified approach to classical and quantum KMS theory. Rep. Math. Phys. (to appear)

9a. Winnink, M.: Algebraic consequences of the KMS boundary condition. In: Cargèse Lectures in Physics, Vol. 4. Kastler, D. (ed.). New York: Gordon and Breach 1970

9b. Winnink, M.: Some general properties of thermodynamic states in an algebraic approach. Statistical mechanics and field theory. Jerusalem, London: Israel University Press 1972

10. Bratteli, O., Robinson, D.W.: Operator algebras and quantum statistical mechanics. I. Chap. 2, Sect. 6. Berlin, Heidelberg, New York: Springer 1979

11. Brinke, G. ten, Winnink, M.: Spectra of Liouville operators. Commun. Math. Phys. 51, 135-150 (1976)

12. Takesaki, M., Winnink, M.: Local normality in quantum statistical mechanics. Commun. Math. Phys. 30, 129-152 (1973)

13. Kastler, D. : Equilibrium states of matter and operator algebras. Proceedings of the Meeting on $C^{*}$ algebras and their Applications to Theoretical Physics. Rome (1975) 
14. Jadczyk, A.Z.: On some groups of automorphisms of von Neumann algebras with a cyclic and separating vector. Commun. Math. Phys. 13, 142-153 (1969)

15. Doplicher, S., Kadison, R.V., Kastler, D., Robinson, D.W.: Asymptotically Abelian systems. Commun. Math. Phys. 6, 101-120 (1967)

16. Gallavotti, G., Verboven, E. : On the classical KMS boundary condition. Nuovo Cimento 28B, 274 (1975)

Communicated by J. Lebowitz

Received August 24, 1981 